Research in Astron. Astrophys. Vol.0 (20xx) No.0, 000-000

http://www.raa-journal.org http://www.iop.org/journals/raa

Research in

(LTEX: zahra'hip101227'v2.tex; printed on November 25, 2021;

$\boldsymbol{A}$ stronomy and

$1: 36)$

Astrophysics

\title{
The Stellar System HIP 101227: Is it a Binary, a Triple or a Quadruple System?
}

\author{
Z. T. Yousef ${ }^{1}$ A. Annuar, ${ }^{1 \star}$ A. M. Hussein ${ }^{2}$, H. M. Al-Naimiy ${ }^{3,4}$, M. A. Al-Wardat, ${ }^{3,4 \star \star}$ N. \\ S. A. Hamid ${ }^{1}$, M. F. Talafha ${ }^{3,4}$ \\ ${ }^{1}$ Department of Applied Physics, Faculty of Science and Technology, Universiti Kebangsaan \\ Malaysia, 43600 UKM Bangi, Selangor, Malaysia; \\ 2 Department of Physics, Al al-Bayt University, Mafraq 25113, Jordan \\ 3 Department of Applied Physics and Astronomy, University of Sharjah, P.O.Box 27272 Sharjah, \\ United Arab Emirates \\ 4 Sharjah Academy for Astronomy, Space Sciences and Technology SAASST, University of Sharjah, \\ P.O.Box 27272 Sharjah, United Arab Emirates
}

Received 20xx month day; accepted 20xx month day

\begin{abstract}
In this paper, we present the analysis of the stellar system HIP 101227 to determine the actual number of components in the system, and their properties. We use dynamical modeling and complex spectrophotometric (involving atmospheric modeling) techniques with recent data, to determine the physical properties and orbital solution for the system, respectively, with better accuracy than past studies. Based on our analysis, we found that the system is more consistent with being a quadruple rather than a binary, or a triple system as that suggested by previous studies. The total mass of the system determined from our SED analysis is $3.42 \pm 0.20 M_{\odot}$, which are distributed almost equally between the four stars. The stars are found to be zero-age main sequence stars; i.e., at the last stage of pre-main sequence,with age less than 200 Myr and spectral types K0. All four stars have very similar physical characteristics, suggesting that fragmentation process is the most likely theory for the formation and evolution of the system.
\end{abstract}

Key words: stars: binaries: close - stars: binaries: visual - stars: individual: HIP 101227

\section{INTRODUCTION}

Most of the stars in our Universe are believed to be part of binary or multiple stellar systems (Duquennoy \& Mayor 1992). The study of these objects is crucial for testing the formation and evolutionary models of stars. This is because they can provide us with the direct measurements of stellar masses (e.g., through dynamical modeling), which is the key property that determines the life cycle of stars. The vast majority of these systems discovered so far are close visual binary and multiple stellar systems (CVBMSs). This means that they appear as a single star even with the largest ground-based telescopes due to their very close separation; i.e., $<0.5^{\prime \prime}$ (e.g., Hilditch 2001). This type of stellar systems can only be identified

* Corresponding author e-mail: adlyka@ukm.edu.my (A.A)

$\star \star$ E-mail: malwardat@sharjah.ac.ae 
Table 1: Basic properties of HIP 101227.

\begin{tabular}{llcl}
\hline Properties & Parameters & Value & Reference \\
\hline Position & Right Ascension & $20^{\mathrm{h}} 31^{\mathrm{m}} 07^{\mathrm{s}} .77$ & SIMBAD \\
& Declination & $+33^{\circ} 32^{\prime} 34^{\prime \prime} .45$ & SIMBAD \\
Magnitude [mag] & Visual Magnitude $\left(m_{\mathrm{v}}\right)$ & 8.34 & ESA (1997) \\
& $B-V$ (Johnson) & $0.88 \pm 0.01$ & ESA (1997) \\
& Visual Extinction $\left(A_{\mathrm{v}}\right)$ & 3.71 & Schlafly \& Finkbeiner (2011) \\
& $B$ (Tycho) & $9.45 \pm 0.02$ & Høg et al. (2000) \\
& $V$ (Tycho) & $8.44 \pm 0.01$ & Høg et al. (2000) \\
Parallax $(\pi)[\mathrm{mas}]$ & old & $22.38 \pm 1.16$ & ESA (1997) \\
& new & $20.47 \pm 0.95$ & Van Leeuwen $(2007)$ \\
\hline
\end{tabular}

using high-resolution observational techniques such as speckle interferometry (e.g., Labeyrie 1970 and Balega \& Tikhonov 1977) and adaptive optics.

One of the most common methods used to study the properties of binary stellar systems is photodynamical modeling. In this technique, the physical and orbital properties of the stars are obtained by modeling the light curves of binary systems during eclipse (Borkovits et al. 2016, 2019, Koçak et al. 2020, and Sürgit et al. 2020). This method however, can only be used for eclipsing binary systems, which are relatively rare.

In 2002, Al-Wardat (2002) introduced a novel method to determine the complete physical properties of CVBMSs through spectral energy distributions (SED) modeling; i.e., atmospheric modeling. The data required for this technique are the magnitude differences obtained from speckle interferometry measurements, and color indices. These data are widely accessible, and therefore the technique can be used to study the properties of all types of CVBMSs, including face-on orbit binaries. This method can be used even in the absence of orbital information and spectroscopic data of the systems. These make it an ideal technique to study the properties of these systems. A series of papers that determine the complete set of physical and orbital properties of CVBMSs using this method and dynamical modeling technique developed by Tokovinin (1992), respectively, have been published for many systems (e.g., Al-Wardat 2007, Al-Wardat \& Widyan 2009, Al-Wardat 2009, Al-Wardat 2012, Al-Wardat et al. 2014a, Al-Wardat et al. 2014b, Al-Wardat et al. 2016, Masda et al. 2016, Al-Wardat et al. 2017, Masda et al. 2018, Masda et al. 2019b and Masda et al. 2019a). These analytical papers aim at taking the advantage of CVBMSs in estimating the key properties of the stellar components which is crucial in understanding the formation and evolution of the systems.

In this paper, we will present the SED and orbital modelings of the triple stellar system candidate HIP 101227 (Tokovinin 2018; Dommanget \& Nys 2002). This system is part of our study of the brightest $(V \leq 10 \mathrm{mag})$ and closest $(d \leq 100 \mathrm{pc})$ triple stellar system candidates in our Universe. Our aim for this project is to determine the true nature of the systems; i.e., whether or not they are indeed triple systems, and to study their physical and orbital properties. These are important towards having a better census on CVBMSs in general.

HIP 101227 is located at a right ascension of $20^{\mathrm{h}} 31^{\mathrm{m}} 07^{\mathrm{s}} .77$, and declination of $+33^{\circ} 32^{\prime} 34^{\prime \prime} .45$ (SIMBAD catalog). The parallax of the system obtained from the Hipparcos New Astrometric Catalog (Van Leeuwen 2007) is $20.47 \pm 0.95$ mas, which corresponds to a distance of $48.85 \pm 0.05 \mathrm{pc}$. In Table 1, we present the basic information for the system. Based on the Multiple Star Catalog by Tokovinin (2018) and Catalog of Components of Double \& Multiple Stars by Dommanget \& Nys (2002), the system is suggested to be a triple system. However this has not been confirmed, and the system has only been analyzed as a binary in previous literatures.

Malkov et al. (2012) derived the total mass of the system, assuming that it is a binary, using dynamical, photometric and spectroscopic techniques. The total masses determined from these techniques were $1.03 \pm 0.31 \mathrm{M}_{\odot}, 1.82 \mathrm{M}_{\odot}$, and $0.85 \mathrm{M}_{\odot}$, respectively. The orbit of the system has previously been determined by Docobo \& Ling (2006) using positional measurements obtained from the Fourth Catalog 
Table 2: Magnitude difference measurements $(\Delta m)$ for HIP 101227 in different filters of the visual band.

\begin{tabular}{lccc}
\hline $\begin{array}{l}\text { Filter } \\
(\lambda / \Delta \lambda)\end{array}$ & $\Delta m$ & $\begin{array}{c}\text { Telescope diameter } \\
{[\mathrm{m}]}\end{array}$ & Reference \\
\hline $511 \mathrm{~nm} / 222 \mathrm{~nm}$ & $0.48 \pm 0.12$ & 0.3 & ESA (1997) \\
$503 \mathrm{~nm} / 40 \mathrm{~nm}$ & $0.28 \pm 0.15$ & 3.5 & Horch et al. (2004) \\
$545 \mathrm{~nm} / 30 \mathrm{~nm}$ & $0.41 \pm 0.03$ & 6.0 & Balega et al. (2006) \\
$541 \mathrm{~nm} / 88 \mathrm{~nm}$ & 0.17 & 3.5 & Horch et al. (2008) \\
$550 \mathrm{~nm} / 40 \mathrm{~nm}$ & 0.63 & 3.5 & Horch et al. (2009) \\
\hline
\end{tabular}

of Interferometric Measurements of Binary Star. ${ }^{1}$ Since then, there are several new positional measurements for the system. We will use these new data in this paper to obtain a more accurate orbital solution for the system. In addition, we will also try to determine the true nature of the system; i.e., whether it is a binary or multiple system, and measure the physical properties of each star in the system.

\section{METHODS}

\subsection{SED Modeling}

In this section, we describe the synthetic SED modeling of HIP 101227 system using the Al-Wardat (2002) technique in order to estimate the physical properties of each star in the system; i.e., the effective temperatures $\left(T_{\text {eff }}\right)$, radii $(R)$, gravitational accelerations $(\log g)$, luminosities $(L)$, masses, ages and spectral types $\left(S_{\mathrm{p}}\right)$. We performed the SED modeling by first assuming that the system is a binary, and then a multiple system.

In order to build individual SED models for the system's components, we first calculated the average value for the magnitude difference between the two main components of the system $(\Delta m)$ in the $V$-band. All of the magnitude difference measurements for HIP 101227 in the $V$-band are tabulated in Table 2. The average value calculated from these data is $\Delta m=0.39 \pm 0.16$.

We then used this value along with the total visual magnitude of the system; i.e., $m_{\mathrm{v}}=8.34$ (ESA 1997), in the following equations to get the apparent magnitudes for the two main components of the system; i.e., component $A$ and $B$ :

$$
\begin{aligned}
& m_{v}^{A}=m_{v}+2.5 \log \left(1+10^{-0.4 \Delta m}\right) \\
& m_{v}^{B}=m_{v}^{A}+\Delta m \\
& M_{V}=m_{v}+5-5 \log d-A_{V}
\end{aligned}
$$

where the interstellar extinction $A_{V}=0.0128$, calculated using $E(B-V)=0.004$ (Lallement et al. 2018). We used the apparent individual magnitude values calculated from these equations, along with $T_{\text {eff }}$, mass, $S_{\mathrm{p}}$ and bolometric correction information obtained from Gray (2005) and Lang (1992), to calculate the bolometric magnitude $M_{\mathrm{bol}}, R, \log g$ and $L$ of the two main components using the wellknown equations for main sequence star:

$$
\begin{aligned}
M_{\mathrm{bol}} & =M_{\mathrm{bol}}^{\odot}-2.5 \log \left(\frac{L}{L_{\odot}}\right) \\
\log \left(\frac{R}{R_{\odot}}\right) & =0.5 \log \left(\frac{L}{L_{\odot}}\right)-2 \log \left(\frac{T_{\mathrm{eff}}}{T_{\mathrm{eff}_{\odot}}}\right) \\
\log g & =\log \left(\frac{\mathrm{M}}{\mathrm{M}_{\odot}}\right)-2 \log \left(\frac{R}{R_{\odot}}\right)+4.43
\end{aligned}
$$

1 The Fourth Catalog of Interferometric Measurements of Binary Stars can be downloaded at http: / / www . astro.gsu . edu/wds/int4/int4_20.html 


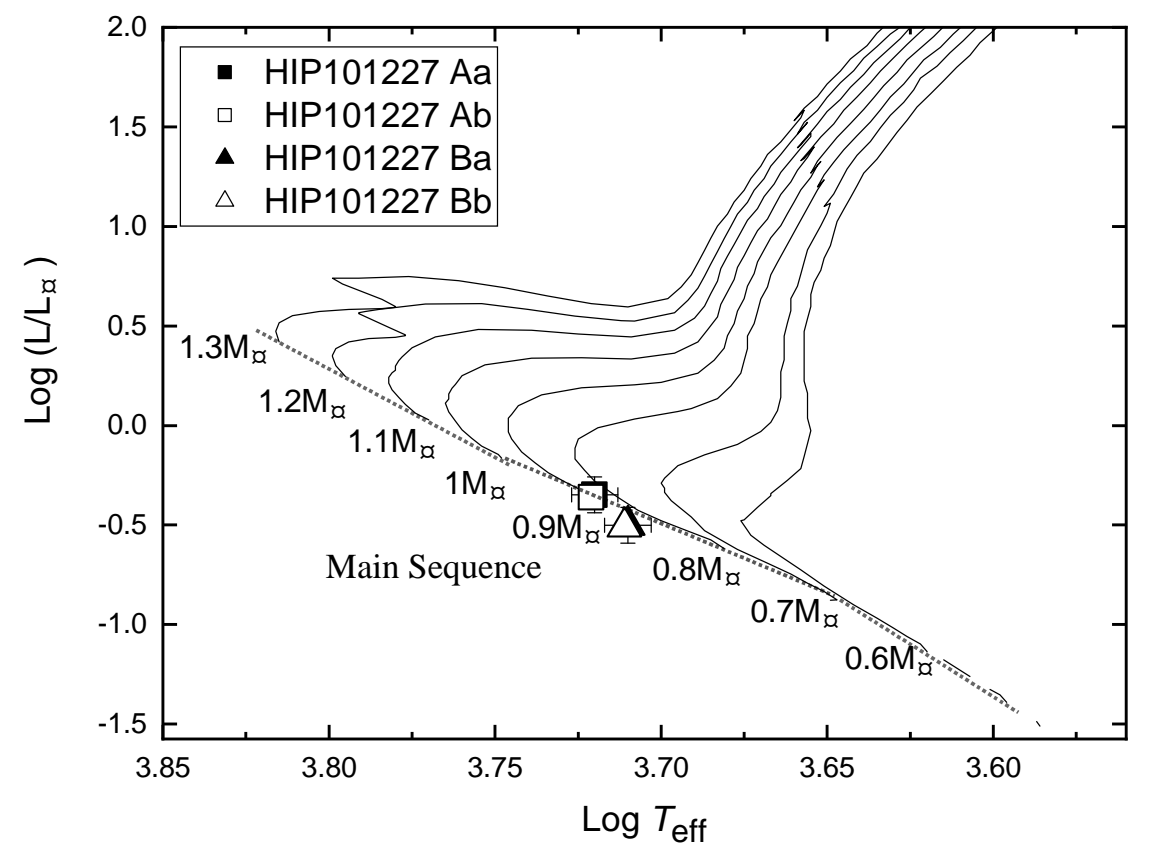

Fig. 1: The positions of each star in the HIP 101227 system on the evolutionary tracks derived by Girardi et al. (2000) assuming that it is a quadruple.

Next, we used these estimated values as preliminary input parameters for the grids of Kurucz's line-blanketed plane-parallel models (ATLAS9) to obtain preliminary synthetic SED for stars $A$ and $B$. These were then combined to get the total synthetic SED of the system according to the following equation (Al-Wardat 2012):

$$
F_{\lambda} \cdot d^{2}=H_{\lambda}^{A} \cdot R_{A}^{2}+H_{\lambda}^{B} \cdot R_{B}^{2}
$$

which can be written as:

$$
F_{\lambda}=\left(R_{A} / d\right)^{2}\left(H_{\lambda}^{A}+H_{\lambda}^{B}\left(R_{A} / R_{B}\right)^{2}\right)
$$

where $H_{\lambda}^{A}$ and $H_{\lambda}^{B}$ represent the flux from a unit surface of the system's components $A$ and $B$, respectively, and $F_{\lambda}$ represents the total SED of the entire system.

In order to get the best synthetic SED that will give the most accurate physical properties for the system, we need to perform the above steps in iterative manner using different sets of preliminary input parameters. This should be performed until we obtained synthetic magnitude and color index values that are consistent with that obtained from observations (SIMBAD catalog) within the error values.

The synthetic magnitudes can be calculated from the synthetic SED using the following relationships (Al-Wardat 2002, 2008, 2012 and references therein):

$$
m_{p}=-2.5 \log \frac{\int P_{p}(\lambda) F_{\lambda, s}(\lambda) \lambda d \lambda}{\int P_{p}(\lambda) F_{\lambda, r}(\lambda) \lambda d \lambda}+Z P_{p}
$$

where $m_{p}$ represents the synthetic magnitude of the pass-band $p, P_{p}$ is the dimensionless sensitivity function of the pass-band $p, F_{\lambda, s}(\lambda)$ and $F_{\lambda, r}(\lambda)$ are the synthetic SEDs of the star being studied and the reference star (Vega in this case), respectively. The zero points, $Z$, were taken from Apellániz (2007). 


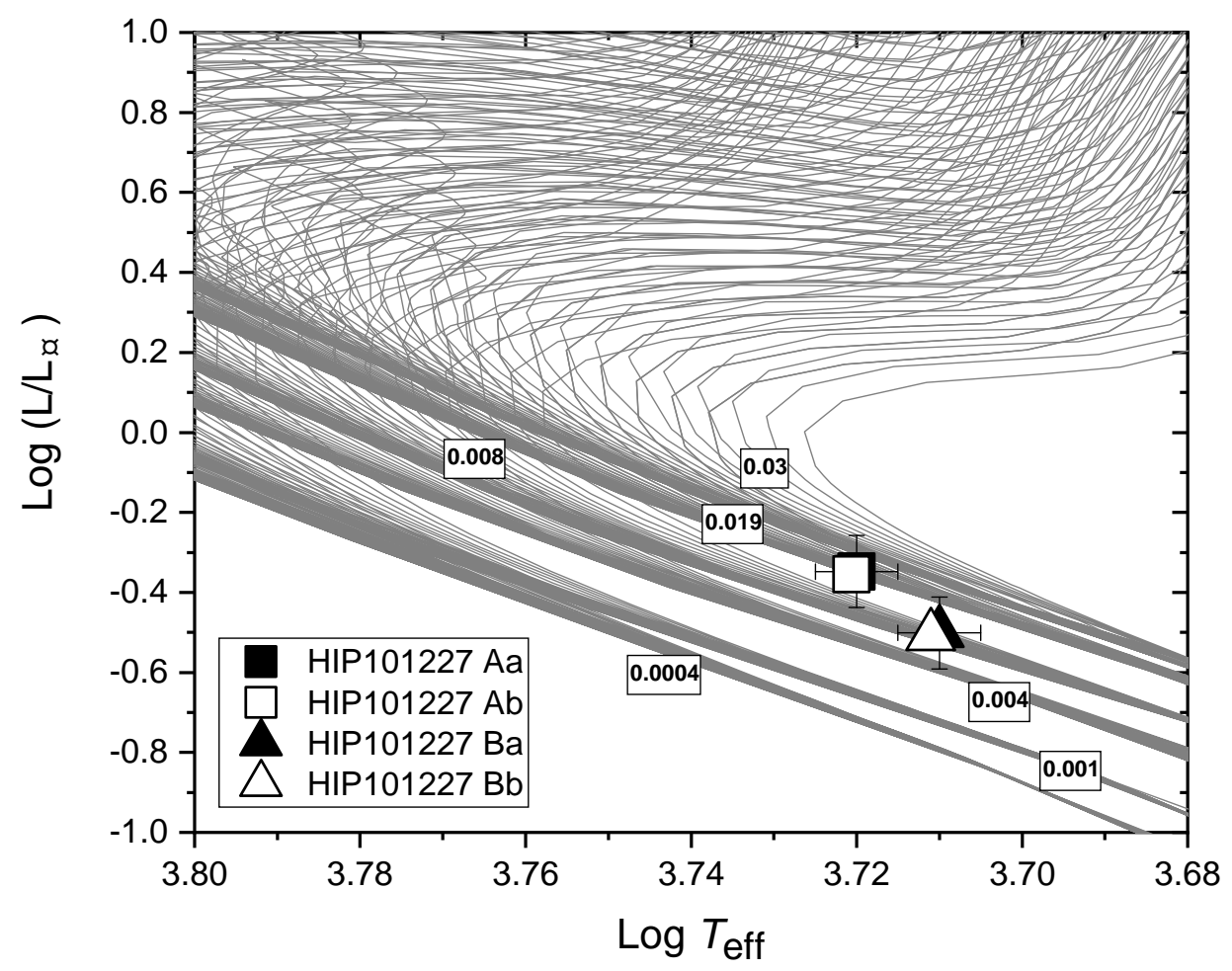

Fig. 2: The positions of each star in the HIP 101227 system on the isochrones for low and intermediatemass stars with different metallicities derived by Girardi et al. (2000) assuming that it is a quadruple.

\subsection{Orbital Analysis}

The orbital properties of HIP 101227 were determined using the dynamical modeling technique developed by Tokovinin (2016) (the original paper is Tokovinin (1992)). This method uses the least-square fits with weights inversely proportional to the observational errors to give the final orbital parameters with their errors using the IDL code ORBIT (Tokovinin 2016) ${ }^{2}$. We used relative position measurements from speckle interferometric observations to determine the period $(P)$, epoch of passage through periastron $\left(T_{\mathrm{o}}\right)$, eccentricity $(e)$, semi-major axis $(a)$, inclination $(i)$, longitude of the periastron $(\omega)$, and the position angle of the line of nodes $(\Omega)$ for the orbit of the two main components of the system.

The code requires the preliminary values of $P, T_{\mathrm{o}}, e, a, i, \omega, \Omega$, the positional measurements, and radial velocities of the system. We obtained positional measurements for the system from the Fourth Catalog of Interferometric Measurements of Binary Stars ${ }^{1}$ and Mason et al. (2018). The latest measurements, which were not available when Docobo \& Ling (2006) did their orbital analysis of the system, are listed in Table 3. We obtained the radial velocities of the whole system from Tabernero et al. (2012) and Gontcharov (2006); i.e., $-23.5 \pm 0.04 \mathrm{~km} \mathrm{~s}^{-1}$ and $-25.6 \pm 0.90 \mathrm{~km} \mathrm{~s}^{-1}$, respectively. The radial velocities for the main components of the system, $A$ and $B$, were obtained from Frasca et al. (2018); i.e., $-31.25 \pm 0.15 \mathrm{~km} \mathrm{~s}^{-1}$ and $-16.88 \pm 0.15 \mathrm{~km} \mathrm{~s}^{-1}$, respectively. We input these values in the ORBIT code to determine the best-fit orbit for the system.

\footnotetext{
2 The IDL code ORBIT can be downloaded at http://www.ctio.noao.edu/ atokovin/orbit/
} 
Table 3: The latest relative positional measurements for the HIP 101227 system (that was not included in Docobo \& Ling 2006) taken from the Fourth Catalog of Interferometric Measurements of Binary Stars. For all positional measurements, please refer to the catalog.

\begin{tabular}{lccccc}
\hline Epoch & Theta $\left(\theta^{\circ}\right)$ & Rho $(\rho)$ & $\lambda(\mathrm{nm})$ & Telescope aperture $(\mathrm{m})$ & Reference \\
\hline 2001.7635 & 139.4 & 0.096 & 541 & 3.5 & Horch et al. (2008) \\
2002.7986 & 153.8 & 0.080 & 600 & 6.0 & Balega et al. (2013) \\
2005.5184 & 60.9 & 0.085 & 520 & 3.5 & Docobo et al. (2008) \\
2006.5223 & 75.5 & 0.099 & 754 & 3.5 & Horch et al. (2008) \\
2006.5223 & 79.1 & 0.112 & 754 & 3.5 & Horch et al. (2008) \\
2007.6018 & 266.0 & 0.144 & 550 & 4.0 & Mason et al. (2018) \\
2007.8197 & 89.9 & 0.149 & 550 & 3.5 & Horch et al. (2010) \\
2008.4563 & 273.4 & 0.166 & 550 & 4.0 & Mason et al. (2018) \\
2008.546 & 97.4 & 0.203 & 530 & 0.7 & Gili \& Prieur (2012) \\
\hline
\end{tabular}

\section{RESULTS AND DISCUSSION}

\subsection{Binary, Triple, or Quadruple?}

Based on the physical properties that were obtained from the best-fit SED of the system as a binary (Table A.1 of the Appendix), we plot the stars on the stellar evolutionary tracks diagram by Girardi et al. (2000). This is shown in Figure A.1 (see Appendix). The figure shows that star $A$ is more evolved than star $B$ even though they have similar masses $\left(\operatorname{mass}_{A}=0.79 \pm 0.20 M_{\odot}\right.$ and $\operatorname{mass}_{B}=0.75 \pm$ $0.20 M_{\odot}$ ). We also plot the stars on the metallicity isochrones by Girardi et al. (2000) (Figure A.3 of the Appendix). Based on the figure, we found that both stars deviate significantly from the isochrones tracks. These results provide evidence that HIP 101227 is likely not a binary system.

Therefore, we proceeded our analysis by assuming that the system is now a triple system as that suggested by Tokovinin (2018) and Dommanget \& Nys (2002). Based on our analysis of the system as a binary, we found that star $A$ has a larger radius than star $B$, thus has a higher probability of consisting of two stars. Hence, we further analyzed star $A$ as a sub-binary system consisting of star $A_{\mathrm{a}}$ and $A_{\mathrm{b}}$, assuming that they have very similar properties (i.e., $\Delta m=0$ ). The synthetic SED for star $A$ represents the total SED for both stars. We repeated our analysis as described earlier in order to build the individual SEDs for the three stellar components, and measure their physical properties.

We then used the results that we obtained (Table A.2 of the Appendix) to plot the stars on the stellar evolutionary and metallicity isochrones diagrams (Figure A.2 and A.4 of the Appendix, respectively) by Girardi et al. (2000). Based on Figure A.4, it can be seen that star B is located away from the isochrones tracks. The positions of the stars in the stellar evolutionary diagram are also not consistent with that expected (stars $A_{\mathrm{a}}$ and $A_{\mathrm{b}}$ are more evolved than star $B$ even though they have similar masses; Mass $A_{a}$ $=\operatorname{Mass}_{A_{b}}=0.90 \pm 0.20 M_{\odot}$ and Mass $\left._{B}=0.75 \pm 0.20 M_{\odot}\right)$. Repeating the same analysis by instead assuming that star $A$ is a single star, and star $B$ is a sub-binary system, we obtained similar results (Table A.3 of the Appendix). These indicate that HIP 101227 is likely not a triple stellar system as well, as that suggested by Tokovinin (2018) and Dommanget \& Nys (2002).

Next, we proceeded by assuming that the system is a quadruple consisting of stars $A_{\mathrm{a}}, A_{\mathrm{b}}, B_{\mathrm{a}}$ and $B_{\mathrm{b}}$. Based on our results, we found that the stars fit very well with the metallicity isochrones tracks shown in Figure 2. The properties of the stars are also consistent with the stellar evolutionary tracks shown in Figure 1. We therefore conclude that HIP 101227 is a quadruple stellar system consisting of stars $A_{\mathrm{a}}, A_{\mathrm{b}}, B_{\mathrm{a}}$ and $B_{\mathrm{b}}$. In Table 4, we present the physical properties of the system as measured from the best-fit SED of the system. The synthetic magnitudes and color indices determined from the best-fit SED is tabulated in Table 5, in comparison with the observed values obtained from the SIMBAD catalog. As can be seen from the table, the synthetic values that we obtained are highly consistent with those measured from observations. We note that there are no significant differences between the total synthetic SEDs that we obtained when assuming that the system is a binary, triple or quadruple system.

Figure 2 indicates that stars $A_{\mathrm{a}}$ and $A_{\mathrm{b}}$ have a metallicity, $Z=0.019$, while star $B_{\mathrm{a}}$ and $B_{\mathrm{b}}$ have $Z=0.008$. The total mass of the system is $3.42 \pm 0.20 M_{\odot}$. This is distributed almost equally 
Table 4: Physical properties of each star in the HIP 101227 quadruple system

\begin{tabular}{lcccc}
\hline Parameters & Comp. $A_{\mathrm{a}}$ & Comp. $A_{\mathrm{b}}$ & Comp. $B_{\mathrm{a}}$ & Comp. $B_{\mathrm{b}}$ \\
$T_{\text {eff }}(\mathrm{K})$ & $5250 \pm 30$ & $5250 \pm 30$ & $5170 \pm 20$ & $5170 \pm 20$ \\
$\mathrm{R}\left(R_{\odot}\right)$ & $0.81 \pm 0.02$ & $0.81 \pm 0.02$ & $0.70 \pm 0.01$ & $0.70 \pm 0.01$ \\
$\log g$ & $4.60 \pm 0.05$ & $4.60 \pm 0.05$ & $4.60 \pm 0.05$ & $4.60 \pm 0.05$ \\
$\mathrm{~L}\left(L_{\odot}\right)$ & $0.45 \pm 0.05$ & $0.45 \pm 0.05$ & $0.31 \pm 0.05$ & $0.31 \pm 0.05$ \\
Mass $\left(M_{\odot}\right)$ & $0.90 \pm 0.20$ & $0.90 \pm 0.20$ & $0.81 \pm 0.20$ & $0.81 \pm 0.20$ \\
\hline
\end{tabular}

Table 5: Comparison between the total magnitudes determined from the best-fit synthetic SED and that obtained from observations (SIMBAD catalog).

\begin{tabular}{lccc}
\hline System & Filter & SIMBAD data base & This work \\
\hline Johnson-Cousins & $\mathrm{B}$ & 9.22 & $9.22 \pm 1.19$ \\
& $\mathrm{~V}$ & 8.34 & $8.34 \pm 1.08$ \\
& $\mathrm{~B}-\mathrm{V}$ & 0.88 & $0.88 \pm 0.11$ \\
Tycho & $B_{T}$ & 9.45 & $9.46 \pm 1.23$ \\
& $V_{T}$ & 8.44 & $8.43 \pm 1.09$ \\
& $B_{T}-V_{T}$ & 1.01 & $1.01 \pm 0.13$ \\
$\Delta m$ & $\ldots$ & 0.39 & $0.39 \pm 0.05$ \\
\hline
\end{tabular}

between the four stars. Based on the temperatures of the stars, they can be classified as type K0 stars. The very similar physical characteristics of the stars in the system, as presented in Table 4, suggest that fragmentation process is the most probable theory for the formation of the system as opposed to capture theory. The latter would usually form a system with relatively different properties; e.g., the masses of the stars would be significantly different. Hierarchical fragmentation during rotational collapse has been suggested to produce binaries and multiple systems (Zinnecker \& Mathieu 2001). This mechanism is possible if the spinning disk around an incipient central proto-star is fragmented, as long as it continues to infall (Bonnell \& Bate 1994).

We can estimate the age of the quadruple system from the isochrones diagram in Figure 2 as follow:

$$
t_{\mathrm{ms}}=f_{\mathrm{ASITR}}\left(m_{A, B}-5 \log d+5-A, Z\right)
$$

where $f_{\mathrm{ASITR}}$ is the age-synthetic isochrones track function and $Z$ is the metallicity of the star. Based on this, we found that the age of the system is less than $200 \mathrm{Myr}$, which means that the four stars are hierarchical zero age main sequence stars (at the last stage of the pre-main sequence). This can also be clearly seen from the positions of the stars on the stellar evolutionary diagram in Figure 1.

\subsubsection{Comparison of the Best-fit Synthetic SED with Observational SED}

In order to test the reliability of our results, which is one of the techniques of Al-Wardat's method, we compare the best-fit total synthetic SED that we determined for the system with its observational SEDs that we obtained specifically for this work, and that firstly obtained by Al-Wardat (2002).

We acquired new observational SED for the system at optical wavelength from the Sharjah Observatory located at Sharjah Academy for Astronomy and Space Sciences in the United Arab Emirates on Julian date 2457201.38422 (one night). Basic Echelle Spectrograph (BACHES) was used with SBIG ST-8300 camera at the Plane Wave Corrected Dall-Kirkham (CDK) 17-inch reflector telescope. $^{3}$ Around 10 spectra were obtained with different exposure times (between $100 \mathrm{~s}$ and $300 \mathrm{~s}$ ). The telescope has a Broad-Band Anti-Reflection (BBAR) multicoated 17" (431.8 mm) aperture, a focal length of $2939 \mathrm{~mm}(\mathrm{f} / 6.8)$ with a Carbon fibre truss design to minimize focus shift with a collimator focal ratio of f/10. The BACHES spectrograph has a spectral range of $392 \mathrm{~nm}$ to $800 \mathrm{~nm}$. A $50 \mu \mathrm{m}$ slit was used, which gives an average spectral resolving power $(R=\lambda / \Delta \lambda)$ of 10000 . Thorium-Argon

\footnotetext{
${ }^{3}$ Further details on BACHES can be found at https://academic.oup.com/mnras/article/443/1/158/ 1481885
} 

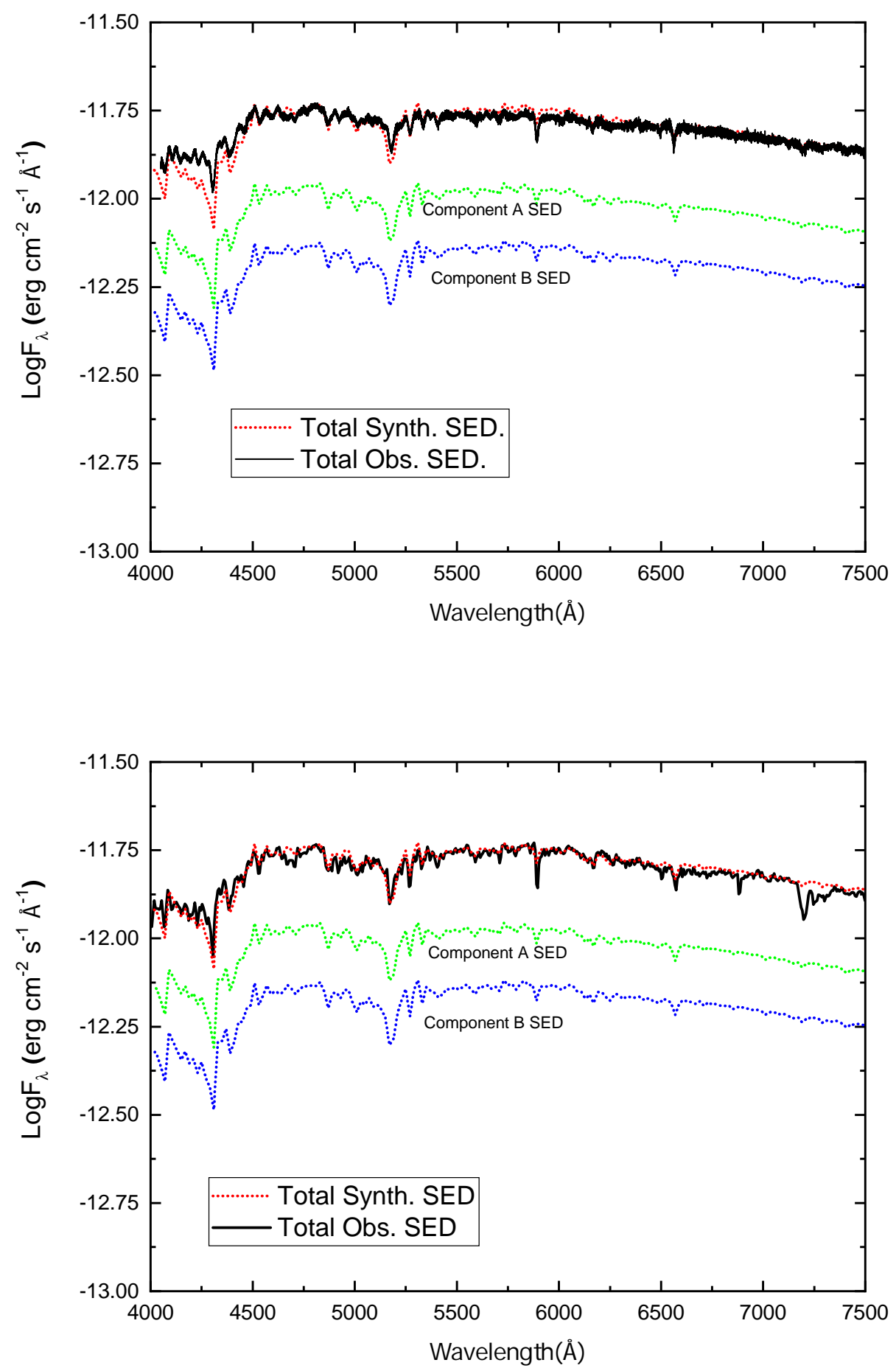

Fig. 3: The best-fit synthetic SEDs for the main stellar components $A$ and $B$, and the total HIP 101227 system (dashed lines) from our work, in comparison with its total SED obtained from our new observations (solid lines) at Sharjah Observatory (top) and Al-Wardat (2002) (bottom). 
Table 6: Orbital parameters of HIP 101227 as measured from our analysis in comparison with those obtained by Docobo \& Ling (2006).

\begin{tabular}{lcc}
\hline Parameter & This work & Docobo \& Ling (2006) \\
\hline$P($ yr. $)$ & $20.514 \pm 0.092$ & 33.13 \\
$T_{\text {o }(\text { yr. })}$ & $1999.097 \pm 0.178$ & 1974.57 \\
$e$ (arcsec) & $0.5193 \pm 0.0138$ & 0.19 \\
$i$ (deg) & $0.1989 \pm 0.0038$ & 0.213 \\
$\Omega($ deg $)$ & $115.9 \pm 0.75$ & 71.5 \\
$\omega($ deg $)$ & $307.07 \pm 2.20$ & 25 \\
Mass $M_{\text {Tot }}\left(M_{\odot}\right)$ & $2.18 \pm 0.33$ & 109.9 \\
\hline
\end{tabular}

cathode and Halogen lamp with blue filter was used for calibration and flat-field, respectively. Vega was used as a reference star.

We stacked the spectra together, and the resultant observational SED for the system is shown in Figure 3. This is compared with the best-fit synthetic SED that we obtained earlier. We also do the same comparison with the old observational SED obtained from Al-Wardat (2002) in Figure 3. Based on this figure, we can see that the delbest-fit synthetic SED profiles and continuum that we built for the entire HIP 101227 system are generally consistent with both observational SED's. There is some disagreement between $\sim 4000-4300 \AA$ in our observation. This can be explained by CCD's sensitivity difference between the red and the blue parts of the spectrum. Overall however, these results provide further support for the results that we obtained from our analysis, and the reliability of the Al-Wardat (2002) method in determining the physical properties of CVBMSs.

\subsection{Orbital Properties}

We initially obtained large errors from our orbital modeling of the system using the Tokovinin (2016) method. This is due to the two points from Ismailov (1992) and Hartkopf et al. (2000) which deviate significantly from the other orbital points. We therefore assigned less weightage for the two points in our fit, and managed to get the best-fit solution.

We show our orbital solution in Figure 4, in comparison with that obtained by Docobo \& Ling (2006). Based on this figure, it can be seen that the two orbits are significantly different from each other, and our orbit fits with the observed positional measurements better than Docobo \& Ling (2006). The average residuals for the fit are $-2.77^{\circ}$ and 6.26 mas for $\theta$ and $\rho$, respectively, and the root-mean-square (RMS) are $7.8833^{\circ}$ and 0.02172 mas for $\theta$ and $\rho$, respectively. We tabulate the orbital properties of the system that we obtained from our analysis in Table 6.

We can independently calculate the total mass of the system using the orbital properties that we obtained from this dynamical analysis. This can be determined using Kepler's third law, which is given by:

$$
\operatorname{Mass}_{\text {Tot }}=\left(\operatorname{Mass}_{\mathrm{A}_{\mathrm{a}}}+\operatorname{Mass}_{\mathrm{A}_{\mathrm{b}}}+\operatorname{Mass}_{\mathrm{B}_{\mathrm{a}}}+\operatorname{Mass}_{\mathrm{B}_{\mathrm{b}}}\right) / \mathrm{M}_{\odot}=\left(\mathrm{a}^{3} / \pi^{3} \mathrm{P}^{2}\right)
$$

where the units of $P$ is in years, $a$ and $\pi$ are in arcseconds. Based on eq. (11), we calculated a total dynamical mass of $2.18 \pm 0.33 M_{\odot}$ for the system. This value is significantly smaller than the total mass determined from our SED analysis; i.e., $3.42 \pm 0.20 M_{\odot}$. A reason for this could be due to inaccuracy in the parallax measurement. The same mass can be obtained if the parallax of the system was to be $\approx 17.95$ mas.

We note that in a private communication with J. A. Docobo during the final submission stage of this paper, we were informed that in their work (Docobo and Campo in preparation), they performed orbital modeling for this system using the same data points as ours, with a different method. The results that they obtained are as follows: $P=20.414$ yr., $T_{\mathrm{o}}=1999.240 \mathrm{yr}$., $e=0.5238, a=0.2011$ arcsec, $i=76.03^{\circ}, \Omega=116.69^{\circ}$ and $\omega=307.21^{\circ}$, with RMS of $7.636^{\circ}$ and 0.0221 mas for $\theta$ and $\rho$ respectively. These orbital elements are highly consistent with that determined in our work (Table 6), providing further support to the results that we obtained. 

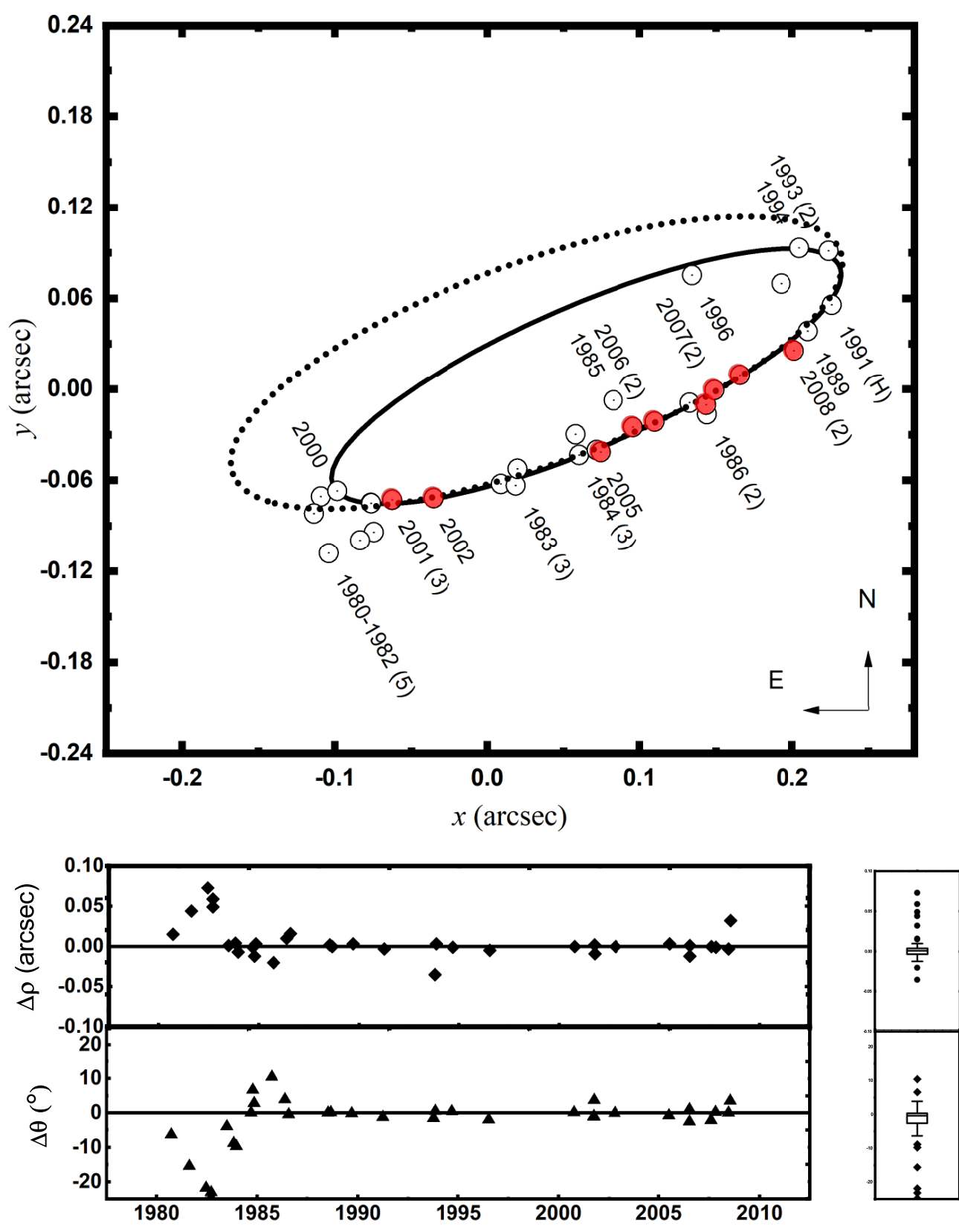

Fig. 4: The orbital solution for HIP 101227 system as determined from our analysis (solid line) and Docobo \& Ling (2006) (dotted line). The white and red circles are the old and new positional points (listed in Table 3), respectively. The bottom left panel show the fit residuals, showing the difference between the observed and model values for the angular separation $(\Delta \rho)$ and position angle $(\Delta \theta)$ of the orbit. The bottom right panel represents the distribution of data based on a five-number summary ("minimum," first quartile (Q1); median, third quartile (Q3); and "maximum") and outliers. 


\section{CONCLUSION}

In this paper, we analyzed the stellar system HIP 101227 to determine the total number of components in the system, and their properties. We used recent data and methods developed by Al-Wardat (2002) (atmospheric modeling) and Tokovinin (2016) (dynamical modeling) to determine the physical properties and orbital solution for the system, respectively, with better accuracy than past studies. Based on our analysis, we found that the system is more consistent with being a quadruple rather than a binary, or a triple system as that suggested by previous studies. This hierarchical quadruple system consists of four zero-age main sequence stars (at the last stage of the pre-main sequence), with age less than 200 Myr and very similar physical properties. Due to their very similar characteristics, we suggested that fragmentation process as the most probable mechanism for the formation of the system.

\section{ACKNOWLEDGMENTS}

We thank the reviewer for his constructive feedback on our manuscript which have helped to significantly improved the paper. We also thank Andrei Tokovinin for his help in solving the orbit of the system, and for valuable discussion.

A. Annuar acknowledges financial support from Malaysia's Ministry of Higher Education Fundamental Research Grant Scheme code FRGS/1/2019/STG02/UKM/02/7. This work has made use of SAO/NASA, SIMBAD database, Fourth Catalog of Interferometric Measurements of Binary Stars, Sixth Catalog of Orbits of Visual Binary Stars, IPAC data systems, ORBITX code and Al-Wardat's complex method for analyzing CVBMSs with its codes, written in FORTRAN and Interactive Data Language (IDL) of the ITT Visual Information Solutions Corporation.

\section{Appendix A: RESULTS FOR BINARY AND TRIPLE SYSTEM ANALYSIS}

Here we provide the results of our analysis when assuming that the system is a binary and triple system, which was discussed in Section 3.1. This includes the physical properties that we determined (Table A.1-A.3), as well as the associated evolutionary track and isochrone diagrams (Figures A.1-A.2 and Figures A.3-A.4, respectively).

Table A.1: Physical properties of each star in the HIP 101227 when assuming that it is a binary system

\begin{tabular}{lcc}
\hline Parameters & Comp. $A$ & Comp. $B$ \\
$T_{\text {eff }}(\mathrm{K})$ & $5250 \pm 30$ & $5170 \pm 20$ \\
$\mathrm{R}\left(R_{\odot}\right)$ & $1.14 \pm 0.01$ & $1.00 \pm 0.01$ \\
$\log g$ & $4.60 \pm 0.05$ & $4.60 \pm 0.05$ \\
$\mathrm{~L}\left(L_{\odot}\right)$ & $0.89 \pm 0.11$ & $0.64 \pm 0.08$ \\
Mass $\left(M_{\odot}\right)$ & $0.79 \pm 0.20$ & $0.75 \pm 0.20$ \\
\hline
\end{tabular}

Table A.2: Physical properties of each star in the HIP 101227 when assuming that it is a triple system, with component $A$ being a sub-binary.

\begin{tabular}{lccc}
\hline Parameters & Comp. $A_{\mathrm{a}}$ & Comp. $A_{\mathrm{b}}$ & Comp. $B$ \\
\hline$T_{\text {eff }}(\mathrm{K})$ & $5250 \pm 30$ & $5250 \pm 30$ & $5170 \pm 20$ \\
$\mathrm{R}\left(R_{\odot}\right)$ & $0.81 \pm 0.02$ & $0.81 \pm 0.02$ & $1.00 \pm 0.01$ \\
$\log g$ & $4.60 \pm 0.05$ & $4.60 \pm 0.05$ & $4.60 \pm 0.05$ \\
$\mathrm{~L}\left(L_{\odot}\right)$ & $0.45 \pm 0.05$ & $0.45 \pm 0.05$ & $0.64 \pm 0.08$ \\
Mass $\left(M_{\odot}\right)$ & $0.90 \pm 0.20$ & $0.90 \pm 0.20$ & $0.75 \pm 0.20$ \\
\hline
\end{tabular}


Table A.3: Physical properties of each star in the HIP 101227 when assuming that it is a triple system, with component $B$ being a sub-binary.

\begin{tabular}{lccc}
\hline Parameters & Comp. $A$ & Comp. $B_{\mathrm{a}}$ & Comp. $B_{\mathrm{b}}$ \\
\hline$T_{\text {eff }}(\mathrm{K})$ & $5250 \pm 30$ & $5170 \pm 20$ & $5170 \pm 20$ \\
$\mathrm{R}\left(R_{\odot}\right)$ & $1.14 \pm 0.01$ & $0.70 \pm 0.01$ & $0.70 \pm 0.01$ \\
$\log g$ & $4.60 \pm 0.05$ & $4.60 \pm 0.05$ & $4.60 \pm 0.05$ \\
$\mathrm{~L}\left(L_{\odot}\right)$ & $0.89 \pm 0.11$ & $0.31 \pm 0.05$ & $0.31 \pm 0.05$ \\
Mass $\left(M_{\odot}\right)$ & $0.79 \pm 0.20$ & $0.81 \pm 0.20$ & $0.81 \pm 0.20$ \\
\hline
\end{tabular}

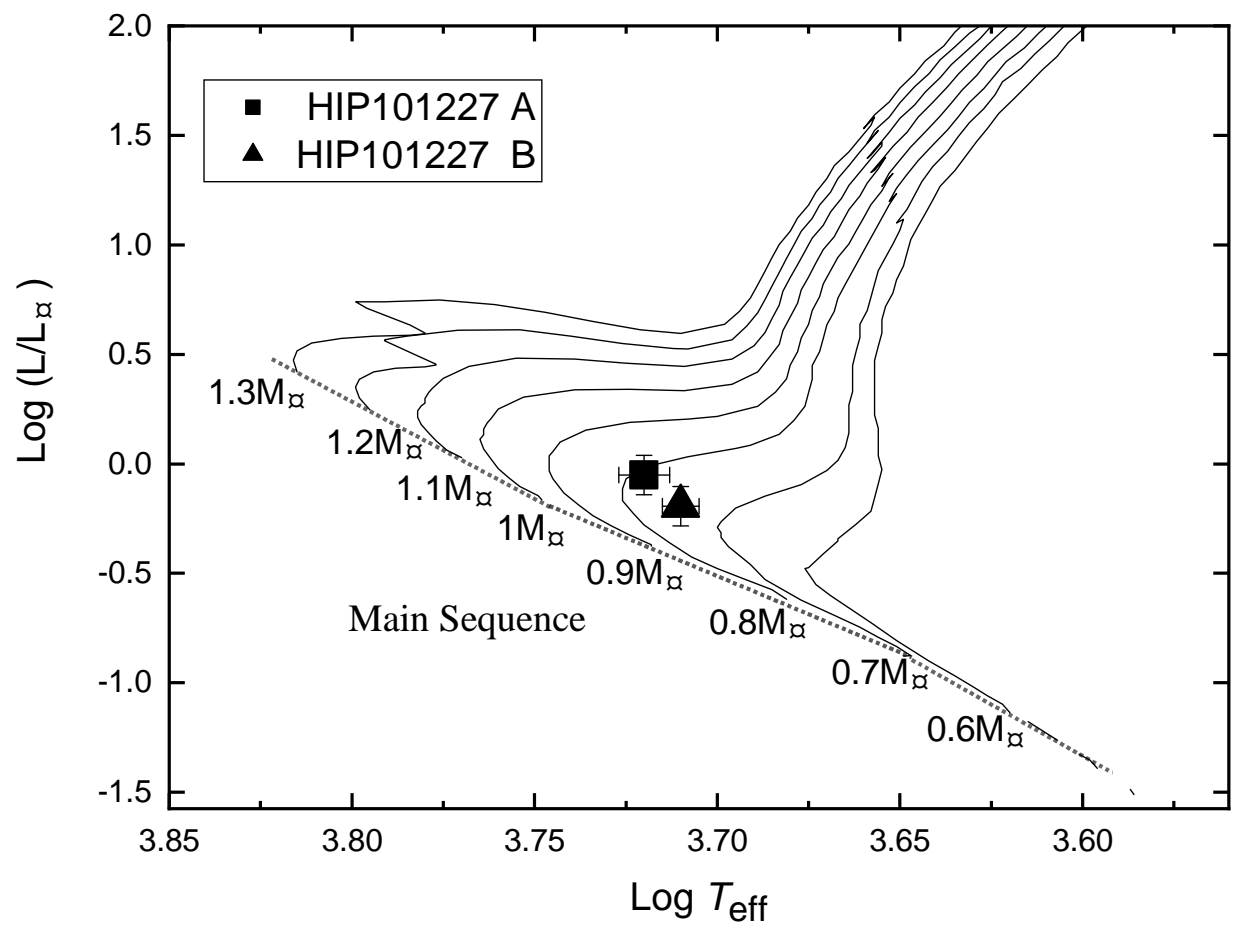

Fig. A.1: The positions of each star in the HIP 101227 system on the evolutionary tracks derived by Girardi et al. (2000) assuming that it is a binary. 

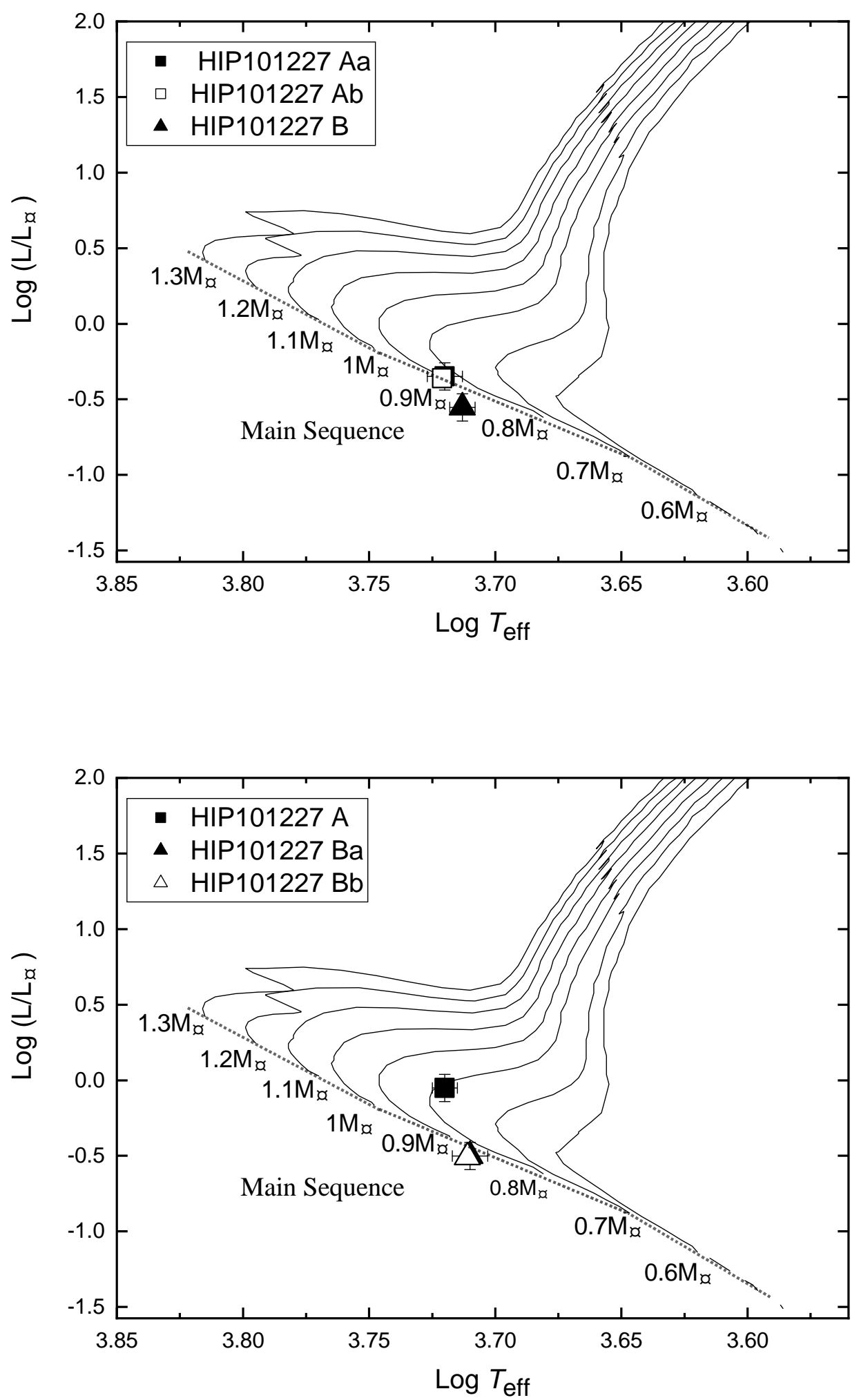

Fig. A.2: The positions of each star in the HIP 101227 system on the evolutionary tracks derived by Girardi et al. (2000) assuming that it is a triple in A (top) and triple in B (bottom). 


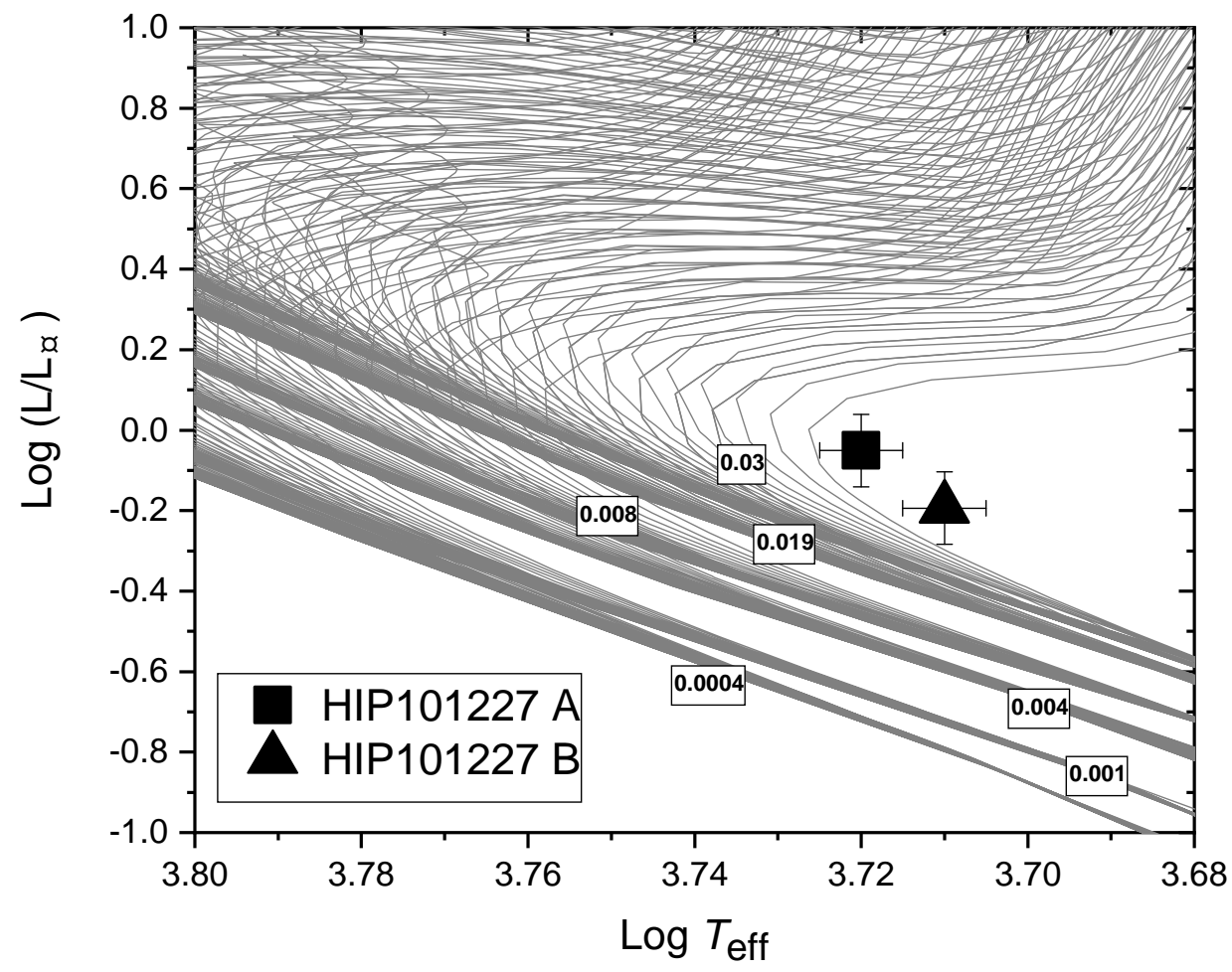

Fig. A.3: The positions of each star in the HIP 101227 system on the isochrones for low and intermediate-mass stars with different metallicities derived by Girardi et al. (2000) assuming that it is a binary. 

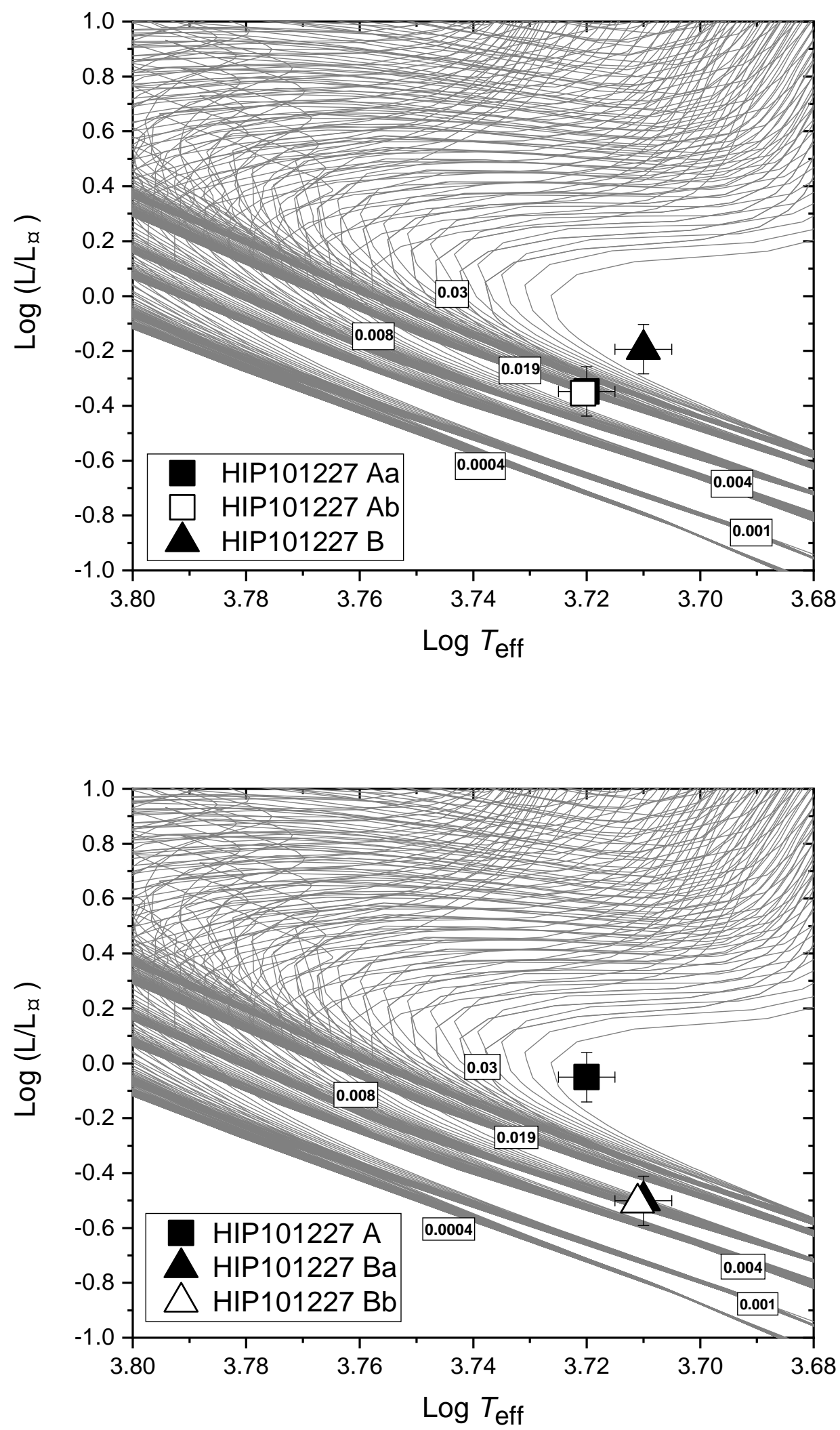

Fig. A.4: The positions of each star in the HIP 101227 system on the isochrones for low and intermediate-mass stars with different metallicities derived by Girardi et al. (2000) assuming that it 


\section{References}

Al-Wardat, M. 2002, Bull. Spec. Astrophys. Obs, 53, 51 2, 3, 4, 7, 8, 9, 11

Al-Wardat, M. 2007, Astronomische Nachrichten: Astronomical Notes, 328, 632

Al-Wardat, M. 2008, Astrophysical Bulletin, 63, 3614

Al-Wardat, M. 2009, Astronomische Nachrichten: Astronomical Notes, 330, 3852

Al-Wardat, M. 2012, Publications of the Astronomical Society of Australia, 29, 523 2, 4

Al-Wardat, M. A., El-Mahameed, M. H., Yusuf, N. A., Khasawneh, A. M., \& Masda, S. G. 2016,

Research in Astronomy and Astrophysics, 16, 1662

Al-Wardat, M., Balega, Y. Y., Leushin, V., et al. 2014a, Astrophysical Bulletin, 69, 582

Al-Wardat, M., Balega, Y. Y., Leushin, V., et al. 2014b, Astrophysical Bulletin, 69, 1982

Al-Wardat, M., Docobo, J., Abushattal, A., \& Campo, P. 2017, Astrophysical Bulletin, 72, 242

Al-Wardat, M., \& Widyan, H. 2009, Astrophysical Bulletin, 64, 3652

Apellániz, J. M. 2007, in the Future of Photometric, Spectrophotometric and Polarimetric Standardization, Vol. 364, 2274

Balega, I., Balega, Y. Y., Maksimov, A., et al. 2006, Bull. Spec. Astrophys. Obs, 59, 203

Balega, I. I., Balega, Y. Y., Gasanova, L. T., et al. 2013, Astrophysical Bulletin, 68, 536

Balega, Y. Y., \& Tikhonov, N. 1977, Soviet Astronomy Letters, 3, 2722

Bonnell, I. A., \& Bate, M. R. 1994, Monthly Notices of the Royal Astronomical Society, 269, L45 7

Borkovits, T., Hajdu, T., Sztakovics, J., et al. 2016, Monthly Notices of the Royal Astronomical Society, 455,41362

Borkovits, T., Rappaport, S., Kaye, T., et al. 2019, Monthly Notices of the Royal Astronomical Society, 483, 19342

Docobo, J. A., Tamazian, V. S., Andrade, M., et al. 2008, AJ, 135, 18036

Docobo, J., \& Ling, J. 2006, IAU Commission on Double Stars, 160 2, 5, 6, 9, 10

Dommanget, J., \& Nys, O. 2002, Observations et Travaux, 54, 5 2, 6

Duquennoy, A., \& Mayor, M. 1992, Binaries as tracers of stellar formation (Cambridge University Press)

ESA. 1997, The Hipparcos and Tycho Catalogues, ESA SP-1200 2, 3

Frasca, A., Guillout, P., Klutsch, A., et al. 2018, A\&A, 612, A96 5

Gili, R., \& Prieur, J.-L. 2012, Astronomische Nachrichten, 333, 7276

Girardi, L., Bressan, A., Bertelli, G., \& Chiosi, C. 2000, Astronomy and Astrophysics Supplement Series, 141, 371 4, 5, 6, 12, 13, 14, 15

Gontcharov, G. A. 2006, Astronomy Letters, 32, 7595

Gray, D. F. 2005, The observation and analysis of stellar photospheres (Cambridge University Press) 3

Hartkopf, W. I., Mason, B. D., McAlister, H. A., et al. 2000, AJ, 119, 30849

Hilditch, R. W. 2001, An introduction to close binary stars (Cambridge University Press) 1

Høg, E., Fabricius, C., Makarov, V. V., et al. 2000, A\&A, 355, L27 2

Horch, E. P., Falta, D., Anderson, L. M., et al. 2009, The Astronomical Journal, 139, 2053

Horch, E. P., Falta, D., Anderson, L. M., et al. 2010, AJ, 139, 2056

Horch, E. P., Meyer, R. D., \& van Altena, W. F. 2004, The Astronomical Journal, 127, 17273

Horch, E. P., van Altena, W. F., Cyr, William M., J., et al. 2008, AJ, 136, 3126

Horch, E. P., Van Altena, W. F., Cyr Jr, W. M., et al. 2008, The Astronomical Journal, 136, 3123

Ismailov, R. M. 1992, A\&AS, 96, 3759

Koçak, D., İçli, T., \& Yakut, K. 2020, arXiv preprint arXiv:2002.05159 2

Labeyrie, A. 1970, Astron. Astrophys., 6, 852

Lallement, R., Capitanio, L., Ruiz-Dern, L., et al. 2018, Astronomy \& Astrophysics, 616, A132 3

Lang, K. 1992, Astrophysical Data: Planets and Stars, 132ff 3

Malkov, O. Y., Tamazian, V., Docobo, J., \& Chulkov, D. 2012, Astronomy \& Astrophysics, 546, A69 2

Masda, S., Docobo, J., Hussein, A., et al. 2019a, Astrophysical Bulletin, 74, 4642

Masda, S. G., Al-Wardat, M. A., Neuhäuser, R., \& Al-Naimiy, H. M. 2016, Research in Astronomy and Astrophysics, 16, 1122 
Masda, S. G., Al-Wardat, M. A., \& Pathan, J. K. M. K. 2018, Research in Astronomy and Astrophysics, 18,0722

Masda, S. G., Al-Wardat, M. A., \& Pathan, J. M. 2019b, Research in Astronomy and Astrophysics, 19, 1052

Mason, B. D., Hartkopf, W. I., Miles, K. N., et al. 2018, AJ, 155, 215 5, 6

Schlafly, E. F., \& Finkbeiner, D. P. 2011, ApJ, 737, 1032

Sürgit, D., Erdem, A., Engelbrecht, C. A., \& Marang, F. 2020, Monthly Notices of the Royal Astronomical Society, 493, 26592

Tabernero, H. M., Montes, D., \& Gonzalez Hernandez, J. I. 2012, VizieR Online Data Catalog, $\mathrm{J} / \mathrm{A}+\mathrm{A} / 547 / \mathrm{A} 135$

Tokovinin, A. 1992, HA McAlister \& WI Hartkopf,(ASP, San Francisco), 32, 573 2, 5

Tokovinin, A. 2016, The Astronomical Journal, 152, 11 5, 9, 11

Tokovinin, A. 2018, ApJS, 235, 6 2, 6

Van Leeuwen, F. 2007, Astronomy \& Astrophysics, 474, 6532

Zinnecker, H., \& Mathieu, R. D. 2001, in Symposium-International Astronomical Union, Vol. 200, Cambridge University Press, 17 